\title{
Clinicopathological findings in patients with primary and secondary defects of neutrophil mobility
}

\author{
A. FARHOUDI,* B. A. M. HARVEY, AND J. F. SOOTHILL \\ From the Department of Immunology, Institute of Child Health, London
}

SUMMARY Twenty-eight patients with defective neutrophil mobility were classified, largely on results of parent values, into primary (17) — mainly Shwachman's syndrome-and probable secondary (11). They presented with frequent infections and/or allergy and these symptoms were essentially similar in both groups. Neutropenia was virtually confined to the patients with Shwachman's syndrome. Diagnoses associated with secondary neutrophil mobility defects included hydrocephalus with shunt, cystic fibrosis, and immunoglobulin deficiency. The neutrophil mobility of nearly all the patients studied increased when levamisole was added in vitro, but there was no clear evidence of clinical benefit when patients were given the drug.

There have been several reports of defects of neutrophil mobility in patients presenting with frequent infection (Miller et al., 1971) or allergy (Hill and Quie, 1974). Sometimes these were associated with recognisable familial syndromes-for example Chediak-Higashi disease (White, 1966), defective actin binding (Boxer et al., 1974), or Shwachman's syndrome (Aggett et al., 1978) but most were sporadic. Neutrophil mobility is influenced by environmental factors, such as infection, and by drugs including corticosteroids (Schär and Meier, 1960).

In any particular patient the only way to establish that defective neutrophil mobility is primary is by detecting a defect in relatives, preferably in symptomless parents. We compared the findings in 28 patients, classified in this way as primary or probable secondary.

\section{Methods}

To measure neutrophil mobility, neutrophils were isolated from heparinised blood by dextran sedimentation, washed in Hanks's medium, and resuspended at $2 \times 10^{8} / \mathrm{ml}$. Chemotactic factor was generated from serum from a healthy donor by Escherichia coli endotoxin (Difco) in Hanks's medium. The mobility of the neutrophils in $0.25 \mathrm{ml}$ of this suspension through millipore membrane of

Received 9 December 1977

*Present address: The Children's Hospital Medical Centre, 62 Bayat Street, Eisenhower Avenue, Tehran, Iran
$3 \mu \mathrm{m}$ pore diameter was measured by the method of Aggett et al. (1978). After 3 hours' incubation the membrane was separated, washed free of red cells, placed in isopropyl-alcohol, washed, stained with haematoxylin, dehydrated, cleared, and mounted on slides. The distance travelled by the front of neutrophils was measured by using a micrometer on the fine adjustment of the microscope ( $\times 40$ objective).

Neutrophil bacterial killing was measured by a modification of the method of Quie et al. (1967). C3 was measured by an immunodiffusion method using whole human serum as standard.

Neutrophil mobility was measured in patients with unexplained frequent infections and in some with allergic disease. Data from a limited control led us to choose the value of $75 \mu \mathrm{m}$ for diagnosing defective mobilities; more data have now led us to reappraise this limit (see below), but it was used for diagnosis in this series. 28 patients gave values below this limit. After observing that there was defective neutrophil mobility in patients with Shwachman's syndrome (Aggett et al., 1978), we included 13 patients with this syndrome and defective mobility. One patient with Shwachman's syndrome in whom the mobility was within the normal range was excluded.

\section{Results}

The results of neutrophil mobility with and without chemotactic stimulation in 48 healthy adults are shown in Fig. 1; values were essentially normally distributed with means $( \pm$ SD) of $109 \cdot 5 \pm 15 \cdot 7 \mu \mathrm{m}$ 


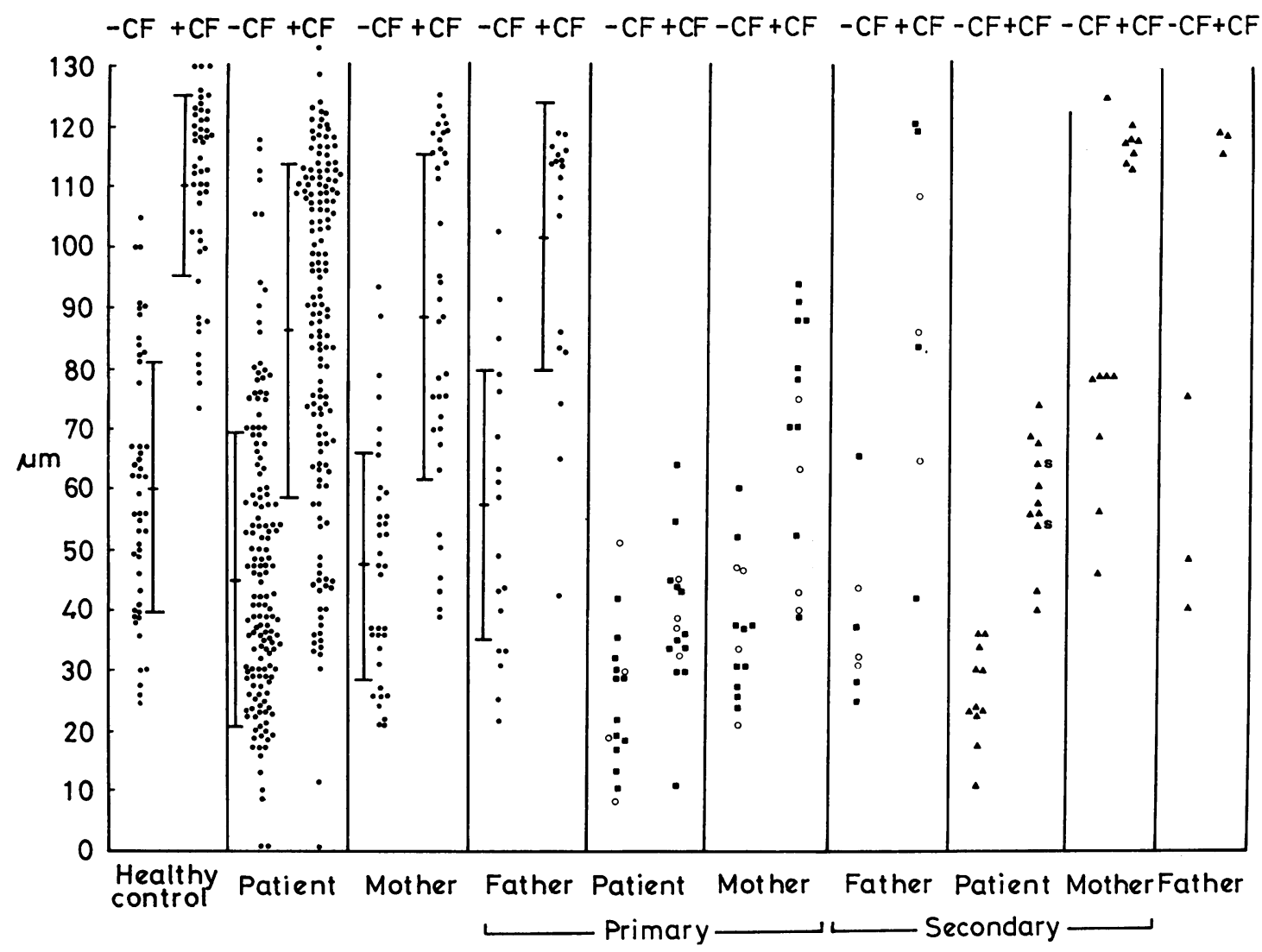

Fig. 1 Leucocyte mobility ( $\mu \mathrm{m})$ with or without chemotactic factor $(+C F,-C F)$ in healthy adults, patients under study, and some of their parents. The patients and parents classified as primary or secondary are plotted separately; Shwachman $(13)=\square$; others, primary $(4)=0$, secondary $(11)=\Delta$.

for the stimulated groups and $60 \pm 21 \cdot 3 \mu \mathrm{m}$ for the unstimulated groups giving lower limits of normal of 78 (stimulated) and $18 \mu \mathrm{m}$ (unstimulated).

A control series of healthy children was not available but the distribution of values in 154 children aged one year or more and tested for diagnostic purposes suggests that there is a similar range in children of this age group to which is added a tail of low values. We also studied 54 of their parents.

We selected 28 children attending The Hospital for Sick Children, for whom full clinical data were available, who gave values of stimulated neutrophil mobility that were lower than our preliminary diagnostic level of $75 \mu \mathrm{m}$.

We measured neutrophil mobility in both healthy parents of 10 patients and in one parent of 11 others. If one or both parents of a patient gave a value $<78 \mu \mathrm{m}$, or if the patient had Shwachman's disease we classified the case as primary. If the parents' values were normal, but there was a possible cause of secondary defect, or if the parents were not tested, the patients were classified as probable secondary.

The results of neutrophil mobility, stimulated and unstimulated, in these groups are shown in Fig. 1. The unstimulated mobility in the patients in both groups and parents in the primary group were also lower than that of the control group (primary patients, $t=6.58 \mathrm{P}<0.0005$; secondary patients $t=5 \cdot 59, \mathrm{P}<0.0005$; primary mothers, $t=3 \cdot 89$, $\mathrm{P}<0.0005$; primary fathers, $t=2 \cdot 61, \mathrm{P}<0.01$ ). The unstimulated values of the parents in the secondary group were not low. We have reported elsewhere that the results in these parents of patients with Shwachman's disease were consistent with manifestation of heterozygosity for an autosomal recessive gene. The 4 patients in the primary group who did not have Shwachman's disease were selected because one or both parents had low values 
of stimulated neutrophil mobility. All had mothers with defective stimulated mobility, and their unstimulated values also tended to be low. One of the 3 fathers studied also gave low values, suggesting an autosomal recessive disorder, but the other 2 were normal; as one patient was a girl, this does not necessarily point to an X-linked inheritance.

Twelve of 17 in the primary group had neutropenia; 11 of these had Shwachman's disease, and one did not. Only one patient (with immunoglobulin deficiency) in the secondary group was neutropenic; there was a significant $(P<0.005)$ difference between the two groups.

The incidence of certain selected clinical features (frequent infection-especially otitis media and skin infection-failure to thrive, diarrhoea, and allergy) in the two groups are shown in Tables 1 and 2. These features which were the reasons for suspecting the diagnosis, were common in both groups; the only significant difference was that diarrhoea was more common in the primary group $(P=0.014)$ but this was largely confined to the patients with Shwachman's disease in whom it is a result of their pancreatic insufficiency (Table 3).

Two patients out of 11 in the secondary group had a Pudenz ventricular shunt for hydrocephalus. Case 25 of the secondary group had several episodes of infections, allergic disorders, and a high level of IgE ( $>6000$ units $/ \mathrm{ml}$ ), as did the patient described by Hill and Quie (1974). But as our patient was being treated with corticosteroids we do not know whether the mobility defect was primary or an effect of the treatment. She, and Case 26, also had defective bacterial killing.

Cases 20, 21, and 22 were siblings, and all had frequent infections and splenomegaly, as did their

Table 1 Clinical and laboratory findings in patients with primary leucocyte mobility defect

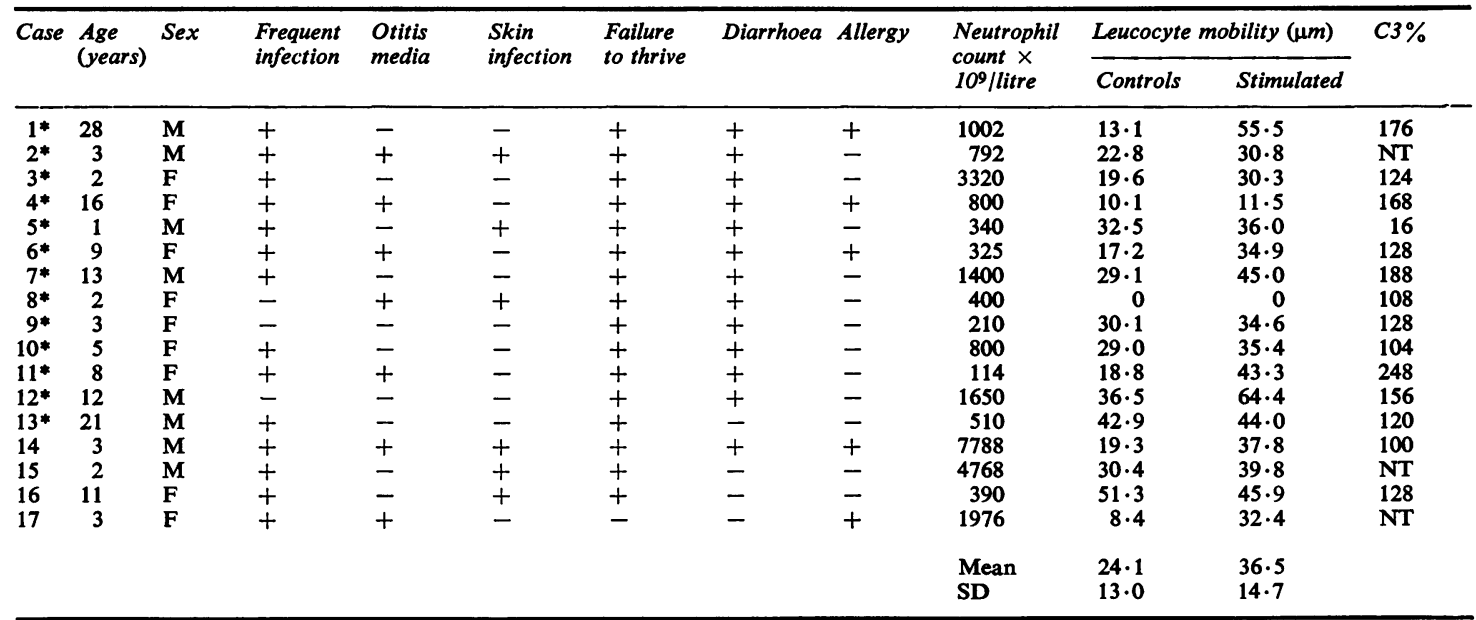

*Shwachman's syndrome. NT = not tested.

Table 2 Clinical and laboratory findings in patients with secondary leucocyte mobility defect

\begin{tabular}{|c|c|c|c|c|c|c|c|c|c|c|c|c|}
\hline \multirow[t]{2}{*}{ Case } & \multirow{2}{*}{$\begin{array}{l}\text { Age } \\
\text { (years) }\end{array}$} & \multirow{2}{*}{ Sex } & \multirow{2}{*}{$\begin{array}{l}\text { Frequent } \\
\text { infection }\end{array}$} & \multirow{2}{*}{$\begin{array}{l}\text { Otitis } \\
\text { media }\end{array}$} & \multirow{2}{*}{$\begin{array}{l}\text { Skin } \\
\text { infection }\end{array}$} & \multirow{2}{*}{$\begin{array}{l}\text { Failure } \\
\text { to thrive }\end{array}$} & \multirow[t]{2}{*}{ Diarrhoea } & \multirow[t]{2}{*}{ Allergy } & \multirow{2}{*}{$\begin{array}{l}\text { Neutrophil } \\
\text { count } \times \\
10^{9} / \text { litre }\end{array}$} & \multicolumn{2}{|c|}{ Leucocyte mobility $(\mu \mathrm{m})$} & \multirow[t]{2}{*}{$C 3 \%$} \\
\hline & & & & & & & & & & Controls & Stimulated & \\
\hline $\begin{array}{l}18 \\
19 \\
20^{*} \\
21^{*} \\
22^{*} \\
23 \\
24 \\
25 \\
26 \\
27 \\
28\end{array}$ & $\begin{array}{l}1 \\
1 \\
3 \\
6 \\
2 \\
1 \\
3 / 4 \\
3 \\
8 \\
1 \\
4\end{array}$ & $\begin{array}{l}\mathbf{M} \\
\mathbf{F} \\
\mathbf{M} \\
\mathbf{F} \\
\mathbf{F} \\
\mathbf{F} \\
\mathbf{F} \\
\mathbf{F} \\
\mathbf{M} \\
\mathbf{M} \\
\mathbf{F}\end{array}$ & $\begin{array}{l}+ \\
+ \\
+ \\
+ \\
- \\
\overline{+} \\
+ \\
+ \\
+ \\
+\end{array}$ & $\begin{array}{l}+ \\
\overline{+} \\
+ \\
- \\
- \\
\overline{+} \\
+ \\
- \\
-\end{array}$ & $\begin{array}{l}+ \\
+ \\
- \\
- \\
- \\
- \\
+ \\
+ \\
+\end{array}$ & $\begin{array}{l}+ \\
+ \\
+ \\
+ \\
- \\
- \\
+ \\
+ \\
+ \\
+\end{array}$ & $\begin{array}{l}+ \\
+ \\
- \\
+ \\
- \\
- \\
- \\
- \\
-\end{array}$ & $\begin{array}{l} \pm \\
- \\
- \\
- \\
- \\
\overline{+} \\
+ \\
-\end{array}$ & $\begin{array}{l}0 \cdot 290 \\
4 \cdot 558 \\
2 \cdot 365 \\
2 \cdot 709 \\
\text { NT } \\
1 \cdot 475 \\
\text { NT } \\
6 \cdot 500 \\
\text { NT } \\
5 \cdot 678 \\
\text { NT } \\
\text { Mean } \\
\text { SD }\end{array}$ & $\begin{array}{r}17 \cdot 4 \\
24 \cdot 8 \\
30 \cdot 8 \\
30.9 \\
36 \cdot 6 \\
36 \cdot 0 \\
22 \cdot 3 \\
34 \cdot 5 \\
11 \cdot 7 \\
23 \cdot 6 \\
29 \cdot 4 \\
\\
27 \cdot 0 \\
7.9\end{array}$ & $\begin{array}{l}40 \cdot 6 \\
43 \cdot 8 \\
67 \cdot 5 \\
60 \cdot 1 \\
56 \cdot 5 \\
64 \cdot 1 \\
57 \cdot 3 \\
74 \cdot 0 \\
56 \cdot 7 \\
54 \cdot 7 \\
68 \cdot 6 \\
58 \cdot 5 \\
10 \cdot 0\end{array}$ & $\begin{array}{r}192 \\
92 \\
92 \\
116 \\
\text { NT } \\
\text { NT } \\
\text { NT } \\
43 \\
96 \\
112 \\
\text { NT }\end{array}$ \\
\hline
\end{tabular}

*Siblings. NT $=$ not tested. 
Table 3 Frequency of certain clinical and laboratory findings in patients with defective neutrophil mobility, primary and secondary

\begin{tabular}{|c|c|c|c|c|}
\hline \multirow{3}{*}{$\begin{array}{l}\text { Clinical and } \\
\text { laboratory findings }\end{array}$} & \multicolumn{4}{|c|}{ Patients with each finding } \\
\hline & \multicolumn{2}{|c|}{ Primary group (17) } & \multicolumn{2}{|c|}{ Secondary group (11) } \\
\hline & $n$ & $\%$ & $n$ & $\%$ \\
\hline Frequent infections & 14 & 82 & 9 & 82 \\
\hline Otitis media & 7 & 41 & 5 & 45 \\
\hline Skin infection & 6 & 35 & 5 & 45 \\
\hline Diarrhoea & 13 & 76 & 3 & 27 \\
\hline Failure to thrive & 16 & 94 & 7 & 64 \\
\hline Allergic disorders & 5 & 29 & 3 & 27 \\
\hline Neutropenia & 12 & 71 & 1 & 9 \\
\hline Immunoglobulin & & & & \\
\hline deficiency & - & - & 2 & 18 \\
\hline Bacterial killing defect & - & - & 2 & 18 \\
\hline
\end{tabular}

mother. Two of them and the mother had had diarrhoea and failure to thrive. We obtained one low mobility value or more in each of the children, but all gave normal values at other times, and the mother's value and that of the healthy father were normal. The clinical state was not obviously related to the value obtained. We cannot be sure whether the mobility abnormality was the cause of this family's troubles or whether both were the effect of another undiagnosed defect.

The qualitative nitroblue tetrazolium (NBT) dye test after in vitro stimulation with $E$. coli endotoxin (presumably a complement mediated stimulus) was normal in all groups. The unstimulated values were above the highest value observed in healthy controls $(12 \%)$ in 9 of the 16 primary patients, and in 5 of their 10 parents. The 3 secondary patients gave raised values but 2 of their parents gave normal results.

When levamisole was added to the medium in vitro at a concentration of $10^{-3} \mathrm{~mol} / 1$, neutrophil mobility was increased in all except 2 of the 13 cases with primary or secondary mobility defects (Fig. 2), but the mobility of polymorphs from 3 healthy subjects was reduced. Some effect was seen at $10^{-4} \mathrm{~mol} / \mathrm{l}$, but none at lower concentrations. The drug was given to one child with primary defect (Case 14) and to 2 with secondary defects (Cases 21 and 26). The mobility of the neutrophils improved in 2 while they were being treated; unstimulated values increased from 13 to 37 , and stimulated values from 28 to 111 ; unstimulated values from 14 to 37 , stimulated values from 47 to 64 , but there was no obvious clinical benefit, and the change of mobility may have been random, or the result of other extraneous factors.

\section{Discussion}

There have been several reports of the association

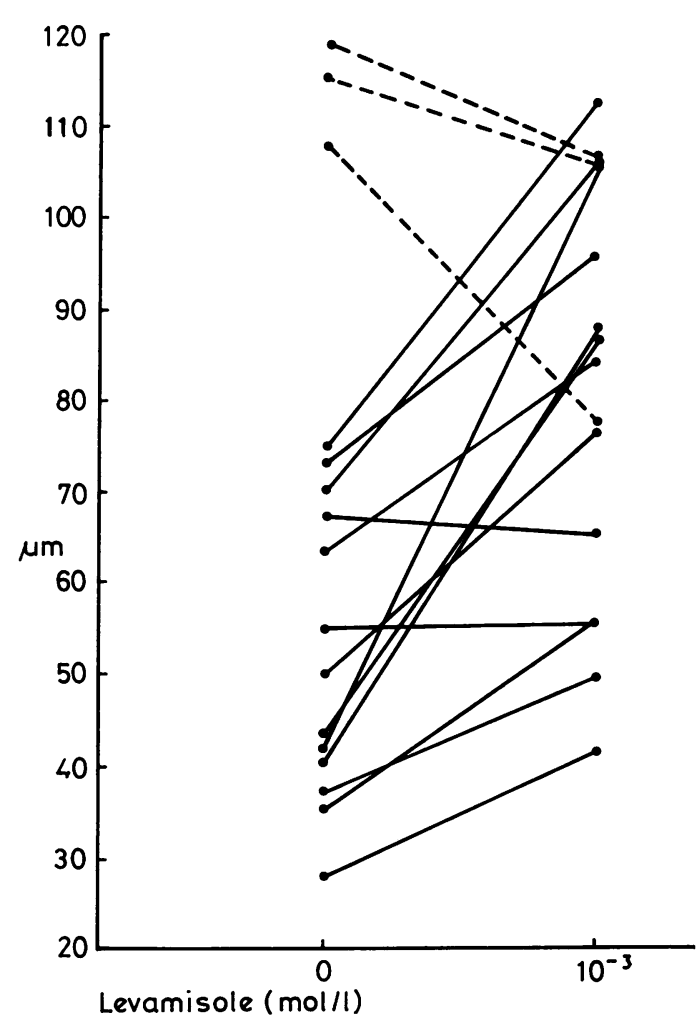

Fig. 2 Mobility ( $\mu m)$ of leucocytes from 13 patients with defective mobility $(\bullet-\bullet)$ and 3 healthy controls $(\bullet---\bullet)$ with and without in vitro incubation with levamisole.

of defective neutrophil mobility with disease, infective and allergic (Hill and Quie, 1974; Blum et al., 1977). In some reports on single cases there is clear evidence of defects in parents (Boxer et al., 1974; Jacobs and Norman, 1977) and consistently within a complex syndrome, Shwachman's disease (Aggett et al., 1978). But leucocyte mobility is very susceptible to environmental factors, so it is difficult to be sure whether the defect is primary or secondary in any particular case without such evidence. The symptoms ascribed to these defects have also been very varied. Some show severe allergy and others not; neutropenia, which has been ascribed to failure of neutrophil migration from marginating pools, is reported in some (Miller et al., 1971) but others with similarly defective neutrophil mobility are not neutropenic. No large series of such patients has systematically been studied. We have therefore analysed the symptoms and laboratory findings of 28 patients with defective neutrophil mobility, classified as primary if one or both parents give low values for neutrophil mobility (most of these are 
Shwachman's disease), or probable secondary if they did not, whether or not a possible cause of the defect was identified.

Diagnosis of patients with such a defect depends on a suitable technique of measurement. Those derived from the membrane passing method of Boyden (1962) are the most widely used, but the many modifications indicate that current methods are unsatisfactory. This is because too many cells are often required for sampling a neutropenic child, the method is not adaptable to many samples, or because of variations in methods for determining the distance travelled through the membrane. The simple technique used in this study has been described elsewhere (Aggett et al., 1978), but we present here data on a population of healthy adults which are consistent with normally distributed data and a lower limit of normal of $78 \mu \mathrm{m}$ is established for this method. The distribution in ill children over oneyear old suggests that similar limits may be applied to them, but leucocyte mobility in babies aged 5 days or less is lower (Miller, 1971) so more data from healthy children are needed before this limit can be fully established.

Our 4 patients, without Shwachman's disease, with defects in one or both parents confirm that there are more than one form of primary neutrophil mobility disorder. The only mechanism demonstrated so far is the defective actin binding shown by Boxer et al. (1974). Jacobs and Norman (1977) described familial association of defective neutrophil mobility with HLA B12. We do not know whether these apply to any of our patients.

Neutropenia was virtually confined to the patients with Shwachman's disease, who have deficiency of other blood cells and evidence of hypoplastic marrow (Shwachman et al., 1964). Many patients with extremely defective neutrophil mobility had normal white counts and even neutrophilia, so we doubt whether slow migration from marginating pools is in fact a cause of neutropenia.

Although we were aware of reports that allergy, especially eczema, was sometimes associated with defective neutrophil mobility (Hill and Quie, 1974), it was an unusual symptom in our series. Diarrhoea and failure to thrive was virtually confined to those with Shwachman's disease, in whom it is caused by the pancreatic insufficiency. We wonder whether some of the patients previously described with mobility defects, neutropenia, diarrhoea, and failure to thrive have, in fact, had Shwachman's syndrome.

Most of the patients classified as probable secondary had no obvious cause, and they may well also have had primary defects. This is probable in the 3 siblings whose mother had a similar syndrome without the defect, but the inconsistency of the mobility defect in these 3 children indicates the difficulty of diagnosing and interpreting such data. We should stress, however, that many of the primary patients have been studied repeatedly and are consistently defective. The association in two patients with specific immunodeficiency (one during a period of transient neutropenia) and in another with cystic fibrosis, may well have been secondary to infection or its treatment. The very low value in one patient who was receiving corticosteroids is consistent with the previous reports that this and other drugs affect the function. Of special interest is the low value in 2 patients with hydrocephalus and Prudenz shunts; it is possible that in vivo activation of the neutrophils by the shunt has rendered them refractory. This could be relevant for the nephritis commonly associated with shunt infection, so we are investigating further the effect of these and other prostheses on this function.

The raised number of NBT-positive cells in unstimulated preparations, compatible with excessive in vivo neutrophil activation, suggests that the surface events initiating polymorph activation occur normally in these patients. Many of the parents of the children in the primary group also had raised values, so the heterozygous state may have some functional significance. The in vitro bacterial phagocytosis and killing was normal in all but two of the secondary group. It seems likely that these two had a different defect from the majority, and that it was probably primary. Mobility and bacterial killing are both defective in Chediak-Higashi disease (White, 1966); these patients do not have this disease.

Most of the patients presented with frequent infections in early childhood. The only one who has died (Case 6) also had gross defects of specific humoral and cellular immunity. Most of the others have improved as they have got older. Boxer et al. (1974) treated their patient with sibling bone marrow graft. We feel that such extreme measures are rarely warranted in these patients. Prophylactic septrin appears to be of value. Although levamisole produces an improvement of neutrophil mobility in vitro and in vivo, we have noticed as have others (Anderson et al., 1976; Wright et al., 1977) no obvious benefit when the drug is given in vivo; a similar in vitro effect of ascorbic acid (Boxer et al., 1974) needs further in vivo appraisal.

\section{References}

Aggett, P. J., Harvey, B. A. M., and Soothill, J. F. (1978). Defective neutrophil mobility in Shwachman's syndrome (in preparation.)

Anderson, R., Glover, A., Koornhof, H. J., and Rabson, A. R. (1976). In vitro stimulation of neutrophil mobility by levamisole. Journal of Immunology, 117, 428-432. 
Blum, R., Geller, G., and Fish, L. H. (1977). Recurrent severe staphylococcal infections, eczematoid rash, extreme elevations of IgF, eosinophilia and divergent chemotactic response in two generations. Journal of Pediatrics, 90, 607-609.

Boxer, L. A., Hedley-Whyte, E. T., and Stossel, T. P. (1974). Neutrophil actin dysfunction and abnormal neutrophil behaviour. New England Journal of Medicine, 291, 10931099.

Boyden, S. V. (1962). The chemotactic effect of mixtures of antibody and antigen on the polymorphonuclear leucocytes. Journal of Experimental Medicine, 115, 453-466.

Hill, H. R., and Quie, P. G. (1974). Raised serum-IgE levels and defective neutrophil chemotaxis in three children with eczema and recurrent bacterial infections. Lancet, 1, 183-187.

Jacobs, J. C., and Norman, M. E. (1977). A familial defect of neutrophil chemotaxis with asthma, eczema, and recurrent skin infections. Pediatric Research, 11, 732-736.

Miller, M. E. (1971). Chemotactic function in the human neonate: humoral and cellular aspects. Pediatric Research, $5,487-492$.

Miller, M. E., Oski, F. A., and Harris, M. B. (1971). Lazy leucocyte syndrome. A new disorder of neutrophil function. Lancet, 1, 665-669.
Quie, P. G., White, J. G., Holmes, B., and Good, R. A. (1967). In vitro bactericidal capacity of human polymorphonuclear leukocytes: diminished activity in chronic granulumatous disease of childhood. Journal of Clinical Investigation, 46, 668-679.

Schär, B., and Meier, R. (1960). Hemmung der durch Proteuslipopolysaccharide gesteigerten Leukozytenemigration in vitro durch antiinflammatorische Corticosteroide. Experientia, 16, 315-316.

Shwachman, H., Diamond, L. K., Oski, F. A., and Khaw, K. T. (1964). The syndrome of pancreatic insufficiency and bone marrow dysfunction. Journal of Pediatrics, 65, 645663.

White, J. G. (1966). Chediak-Higashi syndrome: a possible lysosomal disease. Blood, 28, 143-156.

Wright, D. G., Kirkpatrick, C., and Gallin, J. (1977). Effect of levamisole on normal and abnormal leukocyte locomotion. Journal of Clinical Investigation, 59, 941-950.

Correspondence to Professor J. F. Soothill, Institute of Child Health, Department of Immunology. 30 Guilford Street, London WC1N 1EH. 